\title{
Regional Controllability of Riemann-Liouville Time-Fractional Semilinear Evolution Equations
}

\author{
Asmae Tajani (D), Fatima Zahrae El Alaoui $(\mathbb{D}$, and Ali Boutoulout \\ Department of Mathematics.Faculty of Sciences, Moulay Ismail University, Mekens, Morocco \\ Correspondence should be addressed to Fatima Zahrae El Alaoui; fzelalaoui2011@yahoo.fr
}

Received 6 August 2020; Revised 21 September 2020; Accepted 14 October 2020; Published 4 November 2020

Academic Editor: Xinguang Zhang

Copyright (C) 2020 Asmae Tajani et al. This is an open access article distributed under the Creative Commons Attribution License, which permits unrestricted use, distribution, and reproduction in any medium, provided the original work is properly cited.

In this paper, we discuss the exact regional controllability of fractional evolution equations involving Riemann-Liouville fractional derivative of order $q \in] 0,1$ [. The result is obtained with the help of the theory of fractional calculus, semigroup theory, and Banach fixed-point theorem under several assumptions on the corresponding linear system and the nonlinear term. Finally, some numerical simulations are given to illustrate the obtained result.

\section{Introduction}

Time-fractional systems have been proved, with the development of science and technology, to be one of the most effective tools in modeling many phenomena arising in physics, engineering, and real world problems [1-6]. Therefore, the research studies of fractional-order calculus attract lots of attention for these kinds of systems with several fractional derivatives (for more details, see [7-10] and the references therein). Zhou and Jiao [11] introduced a concept of a mild solution based on Laplace transform and probability density functions; several authors presented a tremendous amount of valuable results on controllability and observability, stability analysis, and so on [12-15].

Similar to the integer-order control systems [16-21], the regional controllability problem of fractional systems is a class of control problems presented in many applications in real world. Regional controllability of linear and some nonlinear fractional systems is referred to in literature [22-24] and the references therein. However, regional controllability of Riemann-Liouville fractional semilinear evolution equations with analytic semigroup problem is still open. Then, this paper focuses on the existence of a bounded control steering the system into a bounded desired state defined only in a subregion of the whole evolution domain. Based on Banach fixed-point theorem and some properties of fractional operators, the main result is deduced.
The rest of this paper is organized as follows. In Section 2 , some definitions and preparation results are introduced. In Section 3, the regional controllability of the considered system, using theory of analytical semigroup, is established under some conditions. At last, two examples are given to illustrate our given algorithm.

\section{Preliminaries and Problem Formulation}

In this section, we introduce some basic definitions of fractional operators present in the considered system which will be specified later and some properties which are used further in this paper.

Definition 1 (see [7]). The left sided Riemann-Liouville fractional integral (resp. derivative) of a function $y$ at a point $t$ of order $q \in$ ]0,1 [ can be written as

$$
\begin{aligned}
\mathrm{I}_{0^{+}}^{q} y(t) & =\frac{1}{\Gamma(q)} \int_{0}^{t}(t-s)^{q-1} y(s) \mathrm{d} s, \quad 0<t \leq T, \\
R L & D_{0^{+}}^{q} y(t)=\frac{\mathrm{d}}{\mathrm{d} t} I_{0^{+}}^{1-q} y(t), \quad 0<t \leq T .
\end{aligned}
$$

Let us consider $X$ and $Y$ to be two Banach spaces; we have the following two propositions. 
Proposition 1 (see [25]). Let us consider $f \in L_{\text {loc }}^{1}(0, T ; X)$ and $T:[0, T] \longrightarrow \mathscr{L}(X, Y)$ to be strongly continuous.

Then, the convolution

$$
(T * f)(t):=\int_{0}^{t} T(t-s) f(s) \mathrm{d} s,
$$

exists in Bochner sense and defines a continuous function $T * f$ from $[0, T]$ into $Y$.

Proposition 2 (see [25]). (Young's inequality).

Let us consider $p, r, s \geq 1$ such that $1 / r+1 / p=1+1 / s$. If $T \in L_{p}(0, T ; \mathscr{L}(X, Y))$ and $f \in L^{r}(0, T ; X)$; then, $T * f \in$ $L^{s}(0, T ; Y)$ and

$$
\|T * f\|_{L^{s}(0, T ; Y)} \leq\|f\|_{L^{r}(0, T ; X)} \cdot\|T\|_{L^{p}(0, T ; \mathscr{L}(X, Y))} .
$$

Now, we present the considered system. For that, let $\Omega$ be a bounded subset of $\mathbb{R}^{n}$ with a smooth boundary $\partial \Omega$. Let us consider $T>0$ and denote

$$
\begin{aligned}
& Q:=\Omega \times] 0, T], \\
& \Sigma:=\partial \Omega \times] 0, T] .
\end{aligned}
$$

We consider the following semilinear fractional system involving Riemann-Liouville derivative of order $q \in] 0,1[$ :

$$
\begin{cases}{ }^{R L} D_{0^{+}}^{q} y(x, t)+A y(x, t)=N y(x, t)+\mathscr{B} u(t), & \text { in } Q, \\ y(\xi, t)=0, & \text { on } \Sigma, \\ \lim _{t \longrightarrow 0^{+}} I_{0^{+}}^{1-q} y(x, t)=y_{0}, & \text { in } \Omega,\end{cases}
$$

where $-A$ is the infinitesimal generator of an analytic semigroup of uniformly bounded operator $\{\mathscr{G}(t)\}_{t \geq 0}$ on the Hilbert space $\mathrm{X}=L^{2}(\Omega)$. Without loss of generality, let $0 \in \rho(A)$ where $\rho(A)$ is the resolvent set of $A$. Then we define the fractional power $A^{\alpha}$ for $0<\alpha<1$, which is a closed linear operator. Its domain is $D\left(A^{\alpha}\right)=\mathrm{X}^{\alpha}$, which is a Banach space equipped with the norm $\|\cdot\|_{X^{\alpha}}=\left\|A^{\alpha}(.)\right\|_{X}, N: L^{2}\left(0, T ; \mathrm{X}^{\alpha}\right) \longrightarrow L^{2}(0, T ; \mathrm{X})$ is a nonlinear operator, and $\mathscr{B}$ is the control operator which is linear (bounded or unbounded) from $\mathbb{R}^{p}$ into $\mathrm{X}$ where $\mathrm{p}$ is the number of actuators, $u$ is given in $U:=L^{2}\left(0, T, \mathbb{R}^{p}\right)$, and the initial state $y_{0}$ is in $\mathrm{X}^{\alpha}$.

We use the following definition of mild solution for the previous problem.

Definition 2 (see [26]). For $t \in] 0, T]$ and any given $u \in U$, we say that a function $y_{u} \in C(0, T ; X)$ is a mild solution of system (6) if it satisfies the following formula:

$$
\begin{aligned}
y_{u}(., t)= & t^{q-1} K_{q}(t) y_{0} \\
& +\int_{0}^{t}(t-\tau)^{q-1} K_{q}(t-\tau)\left(N y_{u}(\tau)+B u(\tau)\right) \mathrm{d} \tau,
\end{aligned}
$$

where

$$
K_{q}(t)=\alpha \int_{0}^{\infty} \theta \phi_{q}(\theta) \mathscr{G}\left(t^{q} \theta\right) \mathrm{d} \theta
$$

in which $\phi_{q}$ is a probability density function defined in ] $0, \infty[$.

Moreover $A^{\alpha}$ and $\mathrm{K}_{q}$ have the following properties.

Proposition 3 (see [27]). For any $t>0$, we have

(i) $\exists M_{\alpha}>0$ such that

$$
\left\|A^{\alpha} K_{q}(t)\right\|_{\mathscr{L}(X, X)} \leq \frac{q M_{\alpha}}{t^{\alpha q}} \times \frac{\Gamma(2-\alpha)}{\Gamma(1+q(1-\alpha))} .
$$

(i) $\exists M>0$ such that

$$
\left\|K_{q}(t)\right\|_{\mathscr{L}\left(X^{\alpha}, X^{\alpha}\right)} \leq \frac{M}{\Gamma(q)} .
$$

Corollary 1. Let us consider

$$
H_{q}(t)=t^{q-1} K_{q}(t)
$$

Then, we have

$$
H_{q} \in L^{1}\left(0, T ; \mathscr{L}\left(X, X^{\alpha}\right)\right) .
$$

Proof. We have $0<\alpha, q<1$; then, $q(1-\alpha)>0$. Therefore, $t^{-\alpha q+q-1} \in L^{1}(0, T)$, and by the previous proposition, we have the result.

For the rest of this paper, we denote

$$
\beta:=\left\|H_{q}\right\|_{L^{1}\left(0, T ; \mathscr{L}\left(X, X^{\alpha}\right)\right)}\|\mathscr{B}\|_{\mathscr{L}\left(X, \mathbb{R}^{p}\right)} .
$$

\section{Regional Controllability}

In this section, we formulate and prove conditions for the regional controllability of semilinear Riemann-Liouville fractional control systems. To do this, let $\omega$ be a subregion of $\Omega$, and we define the restriction operator in $\omega$ by

$$
\begin{aligned}
\chi_{\omega}: L^{2}(\Omega) & \longrightarrow L^{2}(\omega), \\
y & \longrightarrow y_{\left.\right|_{\omega}},
\end{aligned}
$$

and we denote by $\chi_{\omega}^{*}$ its adjoint.

We have the following definition.

Definition 3. The system (6) is said to be exactly (respectively, approximately) $\omega$-controllable if for all $y_{d} \in L^{2}(\omega)$ (respectively, for all $\varepsilon>0$ and for all $y_{d} \in L^{2}(\omega)$ ), there exists a control $u \in U$ such that $\chi_{\omega} y_{u}(T)=y_{d}$ (respectively, $\left.\left\|\chi_{\omega} y_{u}(T)-y_{d}\right\|_{L^{2}(\omega)} \leq \varepsilon\right)$.

For the rest of this paper, we can write the mild solution as follows:

$$
y_{u}(., t)=H_{q}(t) y_{0}+\left(H_{q} * N y_{u}\right)(., t)+\left(H_{q} \star B u\right)(., t),
$$


and we define the restriction of the controllability operator in $\omega$ by

$$
\begin{aligned}
H_{T \omega}^{q}: U & \longrightarrow L^{2}(\omega), \\
u & \longrightarrow \chi_{\omega}\left(H_{q} * B u\right)(., T) .
\end{aligned}
$$

Consider now the following associate linear system of equation (6):

$$
\begin{cases}{ }^{R L} D_{0^{+}}^{q} y_{u}(x, t)=A y_{u}(x, t)+\mathscr{B} u(t), & \text { in } Q, \\ y_{u}(\xi, t)=0, & \text { on } \Sigma, \\ \lim _{t \longrightarrow 0^{+}} I_{0^{+}}^{1-q} y_{u}(x, t)=y_{0}, & \text { in } \Omega,\end{cases}
$$

which we assume to be approximately $\omega$-controllable.

Next, we will study the regional controllability of the system (6) in $I m H_{T \omega}^{q}$ endowed with the norm

$$
\left\|y_{d}\right\|_{\operatorname{Im} H_{T \omega}^{q}}=\left\|H_{T \omega}^{q^{\dagger}} y_{d}\right\|_{U},
$$

where

$$
\left.H_{T \omega}^{q^{\dagger}}:=H_{T \omega}^{q^{*}}\left(H_{T \omega}^{q} H_{T \omega}^{q^{*}}\right)^{-1} \text { (Pseudo-inverse operator of } H_{T \omega}^{q}\right) .
$$

Now, we shall present the main result; we first define the operator

$$
\psi\left(y_{d}, u\right)=H_{T \omega}^{q^{\dagger}}\left(y_{d}-\chi_{\omega} H_{q}(T) y_{0}-\chi_{\omega}\left(H_{q} * N y_{u}\right)(., T)\right),
$$

and we make the following assumptions:

(i) $\left(H_{1}\right)$ For arbitrary $x, y \in L^{2}\left(0, T ; \mathrm{X}^{\alpha}\right)$,

$$
\left\{\begin{array}{l}
N(0)=0, \\
\|N x-N y\|_{L^{2}(0, T ; X)} \leq L_{N}(\|x\|,\|y\|)\|x-y\|_{L^{2}\left(0, T ; X^{\alpha}\right)},
\end{array}\right.
$$

with $L_{N}: \mathbb{R}^{+} \times \mathbb{R}^{+} \longrightarrow \mathbb{R}^{+}$satisfying $\lim _{(a, v) \longrightarrow(0,0)}$ $L_{N}(a, v)=0$.

(ii) $\left(H_{2}\right) \quad\left\|\chi_{\omega} H_{q}(.)\right\|_{\mathscr{L}\left(X, \operatorname{Im} H_{T \omega}^{q}\right)}:=g_{q} \in L^{2}(0, T)$.

We obtain the following theorem.

Theorem 1. If the hypotheses $\left(H_{1}\right)$ and $\left(H_{2}\right)$ are satisfied, then the following assertions hold:

(1) There exist $\rho>0, \mu=\mu(\rho)>0$, and $m=m(\rho)>0$ such that, under the assumption

$$
\rho>(1+\beta) \frac{M T}{\Gamma(q)}\left\|y_{0}\right\|_{X^{\alpha}}
$$

for any state $y_{d}$ in $B(0, \mu) \subset \operatorname{Im} H_{T \omega}^{q}$, the operator $\Psi\left(y_{d},.\right)$ has a unique fixed point $u^{*}$ in $B(0, m)$ that steers system (6) to $y_{d}$ in $\omega$.

(2) The mapping

$$
\begin{aligned}
F: B(0, \mu) & \longrightarrow U, \\
y_{d} & \longrightarrow u^{*}
\end{aligned}
$$

is a Lipschitz mapping.
Proof

(1) Let us consider

$$
\begin{aligned}
& \mathscr{A}_{1}=\beta\left\|g_{q}\right\|_{L^{2}(0, T)} \sup _{a, v \leq \rho} L_{N}(a, v), \\
& \mathscr{A}_{2}=\left\|H_{q}\right\|_{L^{1}\left(0, T ; \mathscr{L}\left(X, X^{\alpha}\right)\right)} \sup _{a, v \leq \rho} L_{N}(a, v)+\frac{M T}{\rho \Gamma(q)}\left\|y_{0}\right\|_{X^{\alpha}}, \\
& \mathscr{A}_{3}=\left\|H_{q}\right\|_{L^{1}\left(0, T ; \mathscr{L}\left(X, X^{\alpha}\right)\right)} \sup _{a, v \leq \rho} L_{N}(a, v) .
\end{aligned}
$$

Using the limit of $\mathrm{L}_{N}(.$, .) near $(0,0)$, we can see that for $\rho>0$, there exists $l>0$ such that

$$
L_{N}(a, v) \leq l<\frac{1-(1+\beta) M T / \rho \Gamma(q)\left\|y_{0}\right\|_{X^{\alpha}}}{\left\|H_{q}\right\|_{L^{1}\left(0, T ; \mathscr{L}\left(X, X^{\alpha}\right)\right)}+\beta\left\|g_{q}\right\|_{L^{2}(0, T)}}
$$

$$
\forall a, v \leq \rho,
$$

which gives $\mathscr{A}_{1}, \mathscr{A}_{2}, \mathscr{A}_{3}<1$.

Let us consider

$$
\begin{aligned}
m= & \frac{\rho}{\beta}\left(1-\left\|H_{q}\right\|_{L^{1}\left(0, T ; \mathscr{L}\left(X, X^{\alpha}\right)\right)} \sup _{a \leq \rho} L_{N}(a, 0)\right. \\
& \left.-\frac{M T}{\rho \Gamma(q)}\left\|y_{0}\right\|_{X^{\alpha}}\right) .
\end{aligned}
$$

We have $m>0$ and the mapping $f: B(0, m) \longrightarrow$ $B(0, \rho)$ such that

$f(u)=y_{u}$ is a Lipschitz mapping with constant $\beta / 1-\mathscr{A}_{3}$. In fact,

$\left\|H_{q}\right\|_{L^{1}\left(0, T ; \mathscr{L}\left(X, X^{\alpha}\right)\right)} \sup _{a \leq \rho} L_{N}(a, 0)+\frac{M T}{\rho \Gamma(q)}\left\|y_{0}\right\|_{X^{\alpha}} \leq A_{2}<1$,

and hence $m>0$.

To show the Lipschitz condition of the function $f$, we use equation (15), and Corollary 1 , we have for all $u, v \in \mathrm{B}(0, m)$

$\left\|y_{u}-y_{v}\right\|_{L^{2}\left(0, T ; X^{\alpha}\right)}$

$=\left\|\left(H_{q} * N\left(y_{u}-y_{v}\right)\right)(.)+\left(H_{q} \star B(u-v)\right)(.)\right\|_{L^{2}\left(0, T ; X^{\alpha}\right)}$

$\leq\left\|H_{q}\right\|_{L^{1}\left(0, T ; \mathscr{L}\left(X, X^{\alpha}\right)\right)}\left\|N\left(y_{u}-y_{v}\right)\right\|_{L^{2}(0, T ; X)}$

$+\left\|H_{q}\right\|_{L^{1}\left(0, T ; \mathscr{L}\left(X, X^{\alpha}\right)\right)}\|\mathscr{R}\|_{\mathscr{L}\left(X, \mathbb{R}^{p}\right)}\|u-v\|_{U}$.

Therefore, by hypothesis $\left(H_{1}\right)$, we obtain

$\left\|y_{u}-y_{v}\right\|_{L^{2}\left(0, T ; X^{\alpha}\right)} \leq \mathscr{A}_{3}\left\|y_{u}-y_{v}\right\|_{L^{2}\left(0, T ; X^{\alpha}\right)}+\beta\|u-v\|_{U}$,

and hence $f$ is a Lipschitz mapping with constant $\beta / 1-\mathscr{A}_{3}$. 
Now, let $\quad \mu=\rho / \beta\left(1-\left((1+\beta) M T / \rho \Gamma(q)\left\|y_{0}\right\|_{X^{\alpha}}+\right.\right.$ Let us consider $u, v \in B(0, m)$; we have $\left.\left.\mathrm{A}_{1}+\mathrm{A}_{3}\right)\right)>0$ and consider $y_{d} \in B(0, \mu)$; we show that $\Psi\left(y_{d},.\right)$ has a unique fixed point in $B(0, m)$.

$$
\begin{aligned}
\left\|\Psi\left(y_{d}, u\right)-\Psi\left(y_{d}, v\right)\right\|_{U} & =\left\|\int_{0}^{T} \chi_{\omega} H_{q}(T-s)\left(N y_{u}(s)-N y_{v}(s)\right) d s\right\|_{\operatorname{Im} H_{T \omega}^{q}} \\
& \leq\left\|g_{q}\right\|_{L^{2}(0, T)}\left\|N y_{u}-N y_{v}\right\|_{L^{2}(0, T ; X)} \leq\left\|g_{q}\right\|_{L^{2}(0, T)} \sup _{a \leq \rho} L_{N}(a, 0)\left\|y_{u}-y_{v}\right\|_{L^{2}\left(0, T ; X^{\alpha}\right)} \\
& \leq \frac{A_{1}}{\beta}\left\|y_{u}-y_{v}\right\|_{L^{2}\left(0, T ; X^{\alpha}\right)^{2}}
\end{aligned}
$$

Since $f$ is Lipschitz, then

$$
\left\|\Psi\left(y_{d}, u\right)-\Psi\left(y_{d}, v\right)\right\|_{U} \leq \frac{\mathscr{A}_{1}}{1-\mathscr{A}_{3}}\|u-v\|_{U}
$$

For $\mathscr{A}_{4}:=\mathscr{A}_{1} / 1-\mathscr{A}_{3}$, using inequality (15), we have $\mathscr{A}_{4}<1$, and thus $\Psi\left(y_{d},.\right)$ is a strict contraction mapping.
Let us show that

$$
\Psi(B(0, \mu), B(0, m)) \subset B(0, m) .
$$

For that, let $u \in B(0, m)$; then, $y_{u} \in B(0, \rho)$ and

$$
\begin{aligned}
\left\|\Psi\left(y_{d}, u\right)\right\|_{U} & =\left\|y_{d}-\chi_{\omega} H_{q}(T) y_{0}-\int_{0}^{T} \chi_{\omega} H_{q}(T-s) N y_{u}(s) d s\right\|_{\operatorname{Im} H_{T \omega}^{q}} \\
& \leq\left\|y_{d}\right\|_{\operatorname{Im} H_{T \omega}^{q}}+\frac{M T}{\Gamma(q)}\left\|y_{0}\right\|+\left\|g_{q}\right\|_{L^{2}(0, T)}\left\|N y_{u}\right\|_{L^{2}(0, T ; X)} \\
& \leq\left\|y_{d}\right\|_{\operatorname{Im} H_{T \omega}^{q}}+\frac{M T}{\Gamma(q)}\left\|y_{0}\right\|+\left\|g_{q}\right\|_{L^{2}(0, T)} \rho \sup _{a \leq \rho} L_{N}(a, 0) .
\end{aligned}
$$

Therefore, for $y_{d} \in B(0, \mu)$, we have

$$
\left\|\Psi\left(y_{d}, u\right)\right\|_{U} \leq \frac{\rho}{\beta}\left(1-\left\|H_{q}\right\|_{L^{1}\left(0, T ; \mathscr{L}\left(X, X^{\alpha}\right)\right)} \sup _{a \leq \rho} L_{N}(a, 0)-\frac{M T}{\rho \Gamma(q)}\left\|y_{0}\right\|_{X^{\alpha}}\right)=m .
$$

Then, $\Psi(B(0, \mu), B(0, m)) \subset B(0, m)$; finally, we deduce from Banach fixed-point theorem that $\Psi\left(y_{d},.\right)$ admits a unique fixed point $u^{*} \in B(0, m)$. A direct calculation shows us that $u^{*}$ obtained is the solution of the regional controllability problem of system (3).

(2) Let us consider $z_{d}$ and $y_{d}$ in $\mathrm{B}(0, \mu)$; we have

$$
\begin{aligned}
F\left(z_{d}\right)-F\left(y_{d}\right)= & \Psi\left(z_{d}, F\left(z_{d}\right)\right)-\Psi\left(z_{d}, F\left(y_{d}\right)\right) \\
& +\Psi\left(z_{d}, F\left(y_{d}\right)\right)-\Psi\left(y_{d}, F\left(y_{d}\right)\right) .
\end{aligned}
$$

On the other hand,

$$
\begin{aligned}
& \left\|\Psi\left(z_{d}, F\left(z_{d}\right)\right)-\Psi\left(z_{d}, F\left(y_{d}\right)\right)\right\|_{U} \leq \mathscr{A}_{4}\left\|F\left(z_{d}\right)-F\left(y_{d}\right)\right\|_{U}, \\
& \left\|\Psi\left(z_{d}, F\left(y_{d}\right)\right)-\Psi\left(y_{d}, F\left(y_{d}\right)\right)\right\|_{U}=\left\|z_{d}-y_{d}\right\|_{\operatorname{Im} H_{T \omega}^{q}}
\end{aligned}
$$

Then,

$$
\left\|F\left(z_{d}\right)-F\left(y_{d}\right)\right\|_{U} \leq \frac{1}{1-A_{4}}\left\|z_{d}-y_{d}\right\|_{\operatorname{Im} H_{T \omega}^{q}} .
$$

Therefore, $F$ satisfies the Lipschitz condition.

Remark 1. In the case where $\mathscr{B}$ is unbounded, we suppose that $\mathscr{B}$ is an admissible control operator for $H_{q}$ (see [28]), 
and consequently we can demonstrate the same result with a suitable $\beta$.

We give the following proposition.

Proposition 4. The sequence

$$
\left\{\begin{array}{l}
u_{0}=0, \\
u_{n+1}=H_{T \omega}^{q^{\dagger}}\left(y_{d}-\chi_{\omega} H_{q}(T) y_{0}-\chi_{\omega}\left(H_{q} * N y_{u_{n}}\right)(T)\right),
\end{array}\right.
$$

converges in $B(0, m) \subset U$ to $u^{*}$.

Proof. Let us consider $n, k \in \mathbb{N}^{*}$; we have

$$
\left\|u_{n+k}-u_{n}\right\|_{U} \leq \sum_{l=n}^{n+k-1}\left\|u_{l+1}-u_{l}\right\|_{U}
$$

From inequality (31), we obtain

$$
\begin{aligned}
\left\|u_{l+1}-u_{l}\right\|_{U} & =\left\|\Psi\left(y_{d}, u_{l}\right)-\Psi\left(y_{d}, u_{l-1}\right)\right\|_{U} \\
& \leq \mathscr{A}_{4}\left\|u_{l}-u_{l-1}\right\|_{U} \leq \mathscr{A}_{4}^{l}\left\|u_{1}\right\|_{U} .
\end{aligned}
$$

Since $\mathscr{A}_{n \rightarrow+\infty}^{n} \longrightarrow 0, \lim _{n \longrightarrow+\infty}\left\|u_{n+k}-u_{n}\right\|_{U}=0$.

Then, $\left(u_{n}\right)_{n}$ is a Cauchy sequence on $B(0, m)$, and we conclude that $\left(u_{n}\right)_{n}$ converges to $u^{*}$ in $B(0, m)$.
Passing to the limit in (8), we have $u^{*}=\Psi\left(y_{d}, u^{*}\right)$; therefore, $u=u^{*}$ because $\Psi\left(y_{d},.\right)$ has a unique fixed point in $B(0, m)$.

Accordingly, we implement the algorithm as follows.

(i) Step 1

$q, y_{0}$, the actuator, the subregion $\omega, y_{d}$ and $\varepsilon$ small enough.

Choose $r_{1}=y_{d}$.

Calculate $u_{1}=H_{T \omega}^{q \dagger} r_{1}$ and obtain $y_{u_{1}}(., T)$.

(ii) Step 2

$$
\begin{aligned}
& \text { Repeat } \\
& \quad r_{n}=r_{n-1}+\left(y_{d}-\chi_{\omega} y_{u_{n-1}}(., T)\right), n \geq 2 . \\
& \text { Calculate } u_{n}=H_{T \omega}^{q \dagger} r_{n} \text { and obtain } y_{u_{n}}(., T) \text {. } \\
& \text { Until }\left\|\chi_{\omega} y_{u_{n}}(., T)-y_{d}\right\|_{I m \mathrm{H}_{T \omega}^{q}}<\varepsilon \text {. }
\end{aligned}
$$

\section{Numerical Simulations}

In this section, we present two numerical simulations illustrating our theoretical result where the first one is given by using zonal actuator and the second example is given by using a pointwise actuator.

4.1. Zonal Actuator. Let us consider the following one-dimensional fractional system with $q=0.3$.

$$
\left\{\begin{array}{l}
\left.{ }^{R L} D_{0^{+}}^{0.3} y_{u}(x, t)=\frac{\partial^{2} y_{u}(x, t)}{\partial x^{2}}+\sum_{j=1}^{\infty}\left(\left\langle y_{u}, \varphi_{j}\right\rangle\right)^{2} \varphi_{j}(x)+\chi_{D} u(t) \text { in }\right] 0,1[\times] 0,2[, \\
\left.y_{u}(\xi, t)=0 \text { on }\{0,1\} \times\right] 0,2[, \\
\left.\lim _{t \longrightarrow 0^{+}} I_{0^{+}}^{0.7} y_{u}(x, t)=\sin (\pi x) \text { in }\right] 0,1[,
\end{array}\right.
$$

where $\varphi_{j}=\sqrt{2} \sin (j \pi x)$ and $D=[0.4,0.6]$.

The subregion where we search the controllability of the considered system is $\omega=[0.2,0.5]$ and let $y_{d}(x)=12 x^{2}(x-1)(x-0.4)$. By using the previous algorithm, we have the following figures.

In Figure 1, we remark that with the given zonal sensor, we obtain successful results which validate the used method and the previous algorithm; indeed, the desired and estimated final states are very close in the subregion $\omega=$ $[0.2,0.5]$ with the error $\epsilon=3 \times 10^{-6}$ which is very small. Figure 2 presents the evolution of control function which has a transfer cost equal to $\left\|u^{*}\right\|^{2}=0.21$.

4.2. Pointwise Actuator. We consider the following system:

$$
\left\{\begin{array}{l}
\left.{ }^{R L} D_{0^{+}}^{0.5} y_{u}(x, t)=\frac{\partial^{2} y_{u}(x, t)}{\partial x^{2}}+\sum_{j=1}^{\infty}\left|\left\langle y_{u}, \varphi_{j}\right\rangle\right|\left\langle y_{u}, \varphi_{j}\right\rangle \varphi_{j}(x)+\delta_{b} u(t) \text { in }\right] 0,1[\times] 0,2[, \\
\left.y_{u}(\xi, t)=0 \text { on }\{0,1\} \times\right] 0,2[, \\
\left.\lim _{t \rightarrow 0^{+}} I_{0^{+}}^{0.5} y_{u}(x, t)=\sin (\pi x) \text { in }\right] 0,1[.
\end{array}\right.
$$




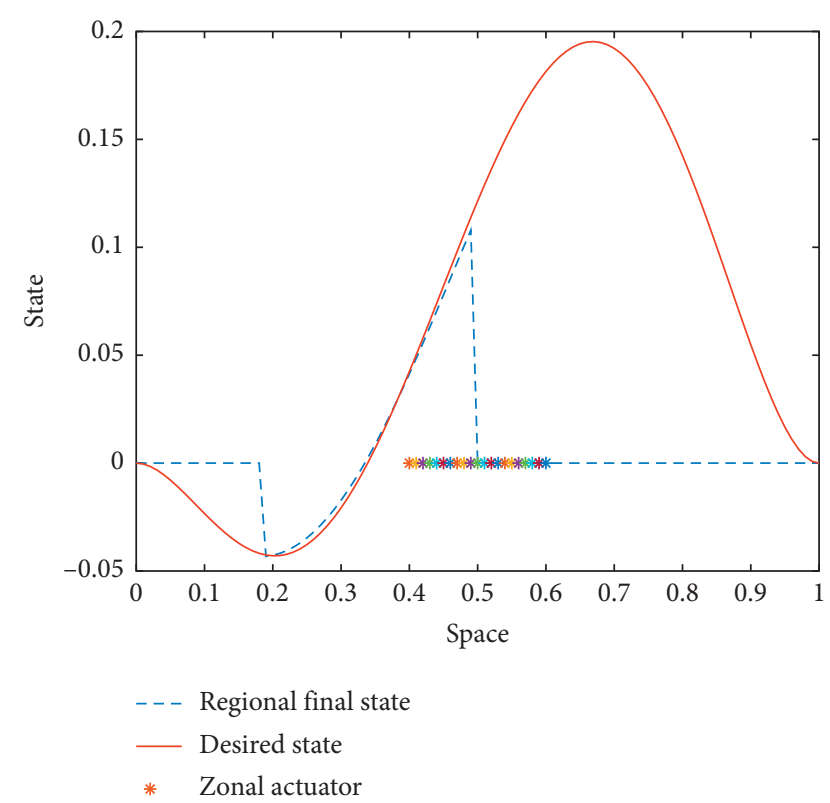

Figure 1: Desired state and estimated final state in $\omega$.

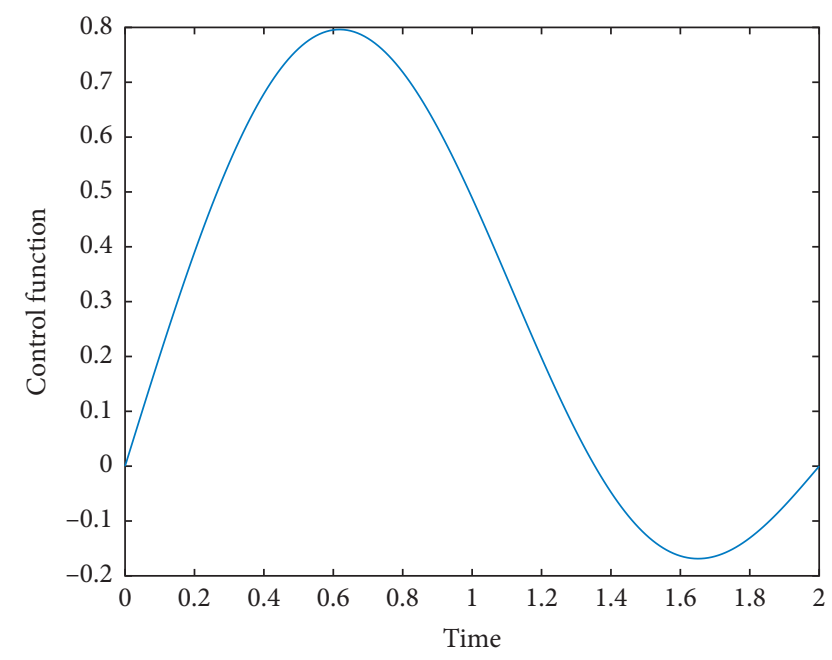

Figure 2: Control input function.

By applying the above algorithm for $b=0.2$, $\omega=[0.35,0.55]$, and

$$
y_{d}(x)=-52 x(x-1)(x-0.33)(x-0.54),
$$

we have the following result.

In Figure 3, like the previous part, the given algorithm leads to good results; we remark that the desired state and the reached one are close in $w=[0.35,0.55]$ using the given pointwise actuator. In this case, the reconstruction error is very small, and it is of order $\varepsilon=10^{-5}$. Figure 4 shows the evolution of control function depending on the time $t$ with the transfer cost $\left\|u^{*}\right\|^{2}=4 \times 10^{-3}$.

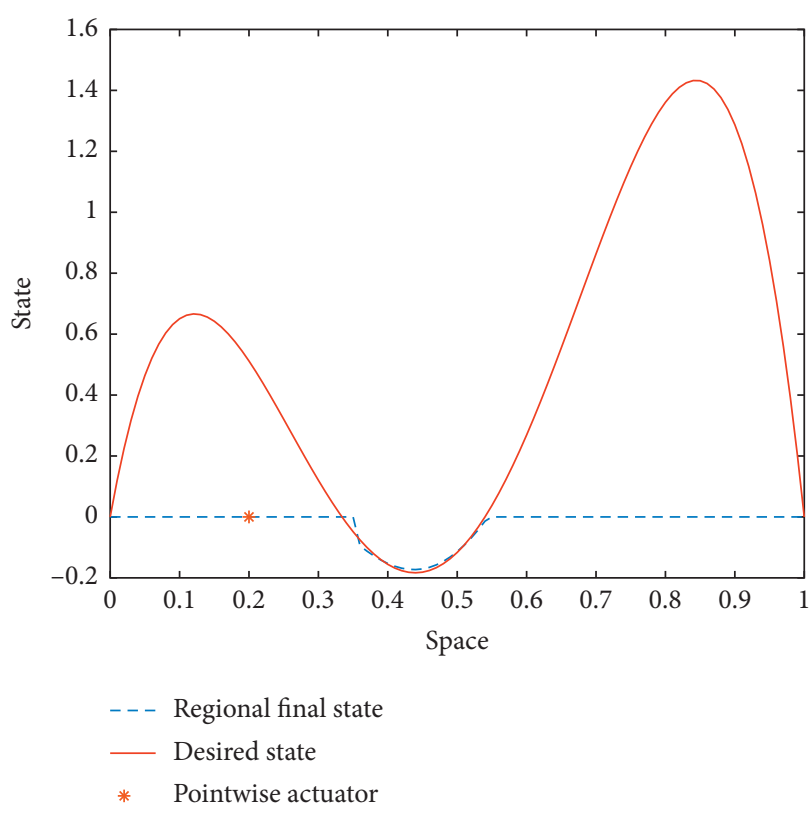

FIgUre 3: Estimated state and desired state in $\omega$.

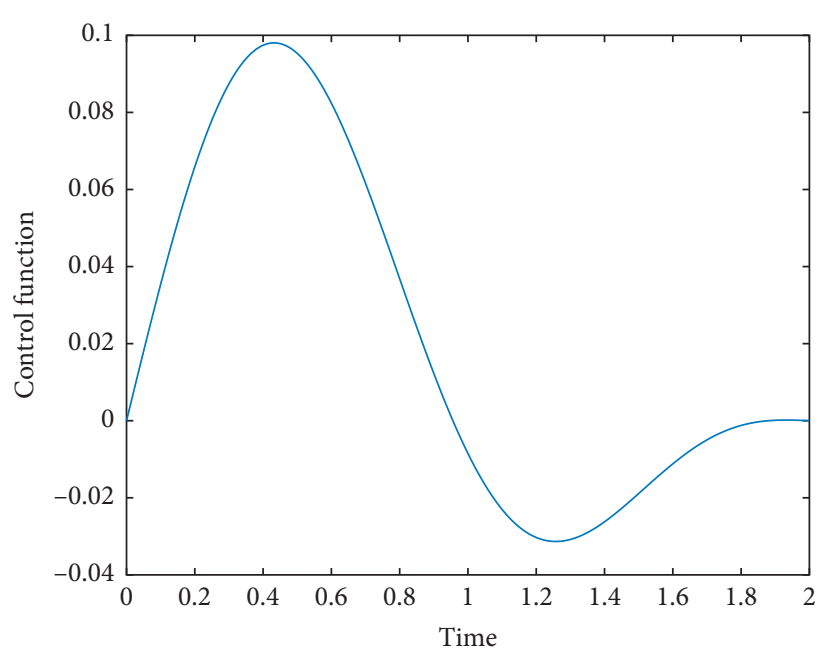

Figure 4: Control function.

\section{Conclusion}

In this paper, we have established the regional controllability for a class of Riemann-Liouville fractional semilinear control systems. The idea of applying control theory for this kind of systems is very interesting and constitutes a new issue in the applications. The presented method in this paper covers a large class of this kind of systems. We have also given an algorithm which has been implemented numerically and has very satisfactory results. In addition, the problem of regional controllability remains open for other types of fractional systems that will be the subject of future research. 


\section{Data Availability}

No data were used to support this study.

\section{Conflicts of Interest}

The authors declare that they have no conflicts of interest.

\section{References}

[1] M. Axtell and M. E. Bise, "Fractional calculus applications in control systems," in Proceedings of the 1990 National Aerospace and Electronics Conference, pp. 563-566, Dayton, $\mathrm{OH}$, USA, May 1990.

[2] R. E. Gutiérrez, J. M. Rosário, and J. Tenreiro Machado, "Fractional order calculus: basic concepts and engineering applications," Mathematical Problems in Engineering, vol. 2010, 19 pages, 2010.

[3] K. Li and J. Peng, "Fractional resolvents and fractional evolution equations," Applied Mathematics Letters, vol. 25, pp. 808-812, 2012.

[4] K. S. Miller and B. Ross, An Introduction to the Fractional Calculus and Fractional Differential Equations, John Wiley \& Sons, New York, NY, USA, 1993.

[5] K. B. Oldham and J. Spanier, Fractional Calculus: Theory and Applications of Differentiation and Integration to Arbitrary Order, Academic Press, Cambridge, London, UK, 1974.

[6] R.-N. Wang, T.-J. Xiao, and J. Liang, "A note on the fractional Cauchy problems with nonlocal initial conditions," Applied Mathematics Letters, vol. 24, no. 8, pp. 1435-1442, 2011.

[7] A. A. Kilbas, H. M. Srivastava, and J. J. Trujillo, Theory and Applications of Fractional Differential Equations, Elsevier, Amsterdam, Netherlands, 2006.

[8] G. Yuchen, S. Xiao-Bao, L. Yougjin, and X. Fei, “The existence and hyers-ulam stability of solution for an impulsive riemann-liouville fractional neutral functional stochastic differential equation with infinite delay of order $1<\beta<2$," Boundary Value Problems, vol. 59, pp. 1-18, 2019.

[9] X. Zhang, L. Liu, and Y. Wu, "Multiple positive solutions of a singular fractional differential equation with negatively perturbed term," Mathematical and Computer Modelling, vol. 55, no. 3-4, pp. 1263-1274, 2012.

[10] X. Zhang, Y. Wu, and L. Caccetta, "Nonlocal fractional order differential equations with changing-sign singular perturbation," Applied Mathematical Modelling, vol. 39, no. 21, pp. 6543-6552, 2015.

[11] Y. Zhou and F. Jiao, "Existence of mild solutions for fractional neutral evolution equations," Computers \& Mathematics with Applications, vol. 59, no. 3, pp. 1063-1077, 2010.

[12] Z. Gao, "Robust stabilization criterion of fractional-order controllers for interval fractional-order plants," Automatica, vol. 61, pp. 9-17, 2015.

[13] T. Karite, A. Boutoulout, and D. F. .M. Torres, "Enlarged controllability of riemann-liouville fractional differential equations," Journal of Computational and Nonlinear Dynamics, vol. 13, Article ID 090907, 2018.

[14] X. Liu, Z. Liu, and M. Bin, "Approximate controllability of impulsive fractional neutral evolution equations with riemann-liouville fractional derivatives," Journal of Computational Analysis and Applications, vol. 17, pp. 468-485, 2014.

[15] H. Zouiten, A. Boutoulout, and D. F. M. Torres, "Regional enlarged observability of Caputo fractional differential equations," Discrete and Continuous Dynamical Systems-Series, vol. 13, no. 3, pp. 1017-1029, 2018.
[16] A. El Jai and A. J. Pritchard, Sensors and Actuators in Distributed Systems Analysis, Ellis Horwood series in Applied mathematics, Wiley, New York, NY, USA, 1988.

[17] T. Karite and A. Boutoulout, "Regional boundary controllability of semi-linear parabolic systems with state constraints," International Journal of Dynamical Systems and Differential Equations, vol. 8, no. 1/2, pp. 150-159, 2018.

[18] J. Klamka, "Constrained controllability of semilinear systems," Nonlinear Analysis: Theory, Methods \& Applications, vol. 47, no. 5, pp. 2939-2949, 2001.

[19] N. I. Mahmudov, "Approximate controllability of semilinear deterministic and stochastic evolution equations in abstract spaces," SIAM Journal on Control and Optimization, vol. 42, no. 5, pp. 1604-1622, 2003.

[20] E. Zerrik, A. Boutoulout, and A. Kamal, "Gradient controllability of parabolic systems," International Journal of Applied Mathematics and Computer Science, vol. 9, no. 4, pp. 767-787, 1999.

[21] E. Zerrik, A. Boutoulout, and A. E. Jai, "Actuators and regional boundary controllability of parabolic systems," International Journal of Systems Science, vol. 31, no. 1, pp. 73-82, 2000.

[22] F. Ge, Y.-Q. Chen, and C. Kou, Regional Analysis of TimeFractional Diffusion Processes, Springer International Publishing, Berlin, Germany, 2018.

[23] S. Linxin, S. Xiao-Bao, and M. Jianzhong, "Approximate controllability and existence of mild solutions for riemannliouville fractional stochastic evolution equations with nonlocal conditions of order $1<\alpha<2$," Fractional Calculus and Applied Analysis, vol. 22, no. 4, pp. 1086-1112, 2019.

[24] A. Tajani, F.-Z. El Alaoui, and A. Boutoulout, "Regional controllability for caputo type semi-linear time-fractional systems," in Proceeding of SM2A'9 Conference on Nonlinear Analysis, Applications and Computational Methods, 2021.

[25] W. Arendt, C. J. K. Batty, M. Hieber, and F. Neubrander, Vector-valued Laplace transforms and Cauchy problems, Birkhäuser, Basel, Switzerland, 2nd edition, 2011.

[26] M. M. El-Borai, "Some probability densities and fundamental solutions of fractional evolution equations," Chaos, Solitons \& Fractals, vol. 14, no. 3, pp. 433-440, 2002.

[27] J. Wang and Y. Zhou, "Analysis of nonlinear fractional control systems in Banach spaces," Nonlinear Analysis: Theory, Methods \& Applications, vol. 74, no. 17, pp. 5929-5942, 2011.

[28] D.-T. Hoang, Controllability And Observability Of non Autonomous Evolution Equations, Optimization and Control, Université de Bordeaux, Bordeaux, France, 2018. 